

\title{
DESENVOLVIMENTO DE METODOLOGIA PARA GERENCIAMENTO DE PROJETO DE REVESTIMENTO DE POÇO DE PETRÓLEO
}

\author{
DEVELOPMENT OF METHODOLOGY FOR WELL CASING PROJECT MANAGEMENT
}

\author{
(iD) Joao Paulo Lima Santos ${ }^{1}$ \\ Eduardo Toledo de Lima Junior ${ }^{2}$ \\ iD Lucas Pereira de Gouveia ${ }^{3}$ \\ Dilvio Ribeiro Dias Junior ${ }^{4}$
}

${ }^{1}$ Doutorado em Engenharia Civil Universidade Federal de Alagoas. Maceió, Alagoas - Brasil. joao.santos@ctec.ufal.br

${ }^{2}$ Doutorado em Engenharia Civil Universidade Federal de Alagoas. Maceió, Alagoas - Brasil. limajunior@lccv.ufal.br

${ }^{3}$ Mestrado em Engenharia Civil Universidade Federal de Alagoas. Maceió, Alagoas - Brasil. lucasgouveia@lccv.ufal.br

${ }^{4}$ Engenheiro de Petróleo Universidade Federal de Alagoas. Maceió, Alagoas - Brasil. silviordjr@outlook.com

Recebido em: 15 ago. 2019

Aprovado em: 13 fev. 2020
Resumo: O presente trabalho propõe-se a desenvolver uma estratégia de otimização de seleção de revestimento de poço combinando a avaliação de integridade mecânica de revestimento por meio de análise triaxial de tensões em estado limite de serviço e técnicas de otimização baseada no mínimo custo global. A formulação é desenvolvida em um sistema de aplicação web (SAEP) construído a partir do cálculo de esforços e cálculo de resistências, que permitem uma análise desacoplada do processo de dimensionamento de coluna de revestimento de poços de petróleo. O módulo de integração e análise permite avaliar de forma integrada as solicitações e a resistência dos tubulares, verificando o atendimento aos critérios de projeto baseado em diretrizes normativas internacionais. A análise da integridade estrutural é realizada através da combinação da envoltória API com a curva limite de plasticidade, auxiliando o usuário no processo de dimensionamento ótimo das colunas de revestimento.

Palavras-chave: Aplicação Web. Dimensionamento de Revestimentos. Otimização. Poços de Petróleo.

Abstract: The present work proposes a strategy for selection of well casing combining triaxial mechanical analysis in serviceability limit state and optimization techniques based on the minimum overall cost. The formulation is developed into an in-house web application system (SAEP), which allow a decoupled analysis of the oil well casing string design. The integration and analysis module allows the integrated evaluation of the applied loads and resistance of the tubulars, verifying the fulfillment of design criteria based on international normative guidelines. Structural integrity analysis is performed by combining the API envelope with the plasticity limit curve, assisting the user in the design of the casing strings.

Keywords: Web Application. Casing Design. Optimization. Oil Well. 
Introdução

A construção de poços de petróleo ocorre por fases, onde, ao final de cada etapa de perfuração, o poço aberto é revestido por uma coluna de aço, em um processo conhecido por revestimento de poço. A etapa de revestimento é uma das mais onerosas e é de extrema importância para a segurança operacional do poço durante sua perfuração, bem como em toda sua vida produtiva. Diante de tais fatos, deve-se procurar garantir que a execução desta etapa seja segura e economicamente satisfatória. Alguns trabalhos indicam que os custos de revestimento de poço podem atingir $50 \%$ do orçamento total, conforme discutido em Correia \& Santos (2017), Ezeakacha et al. (2018), Liu et al. (2018), Aadnoy (2010) e Xia et al. (2015).

Um esquema típico de um programa de revestimento está exemplificado pela Figura 1. 0 revestimento condutor é geralmente o primeiro a ser instalado no poço, com objetivo de garantir integridade nas porções mais superficiais do solo, em conjunto com o revestimento de superfície. O revestimento de produção tem a função de proteger as zonas produtoras no reservatório, enquanto o revestimento intermediário faz a interligação entre as zonas de produção e de superfície, conforme discutido por Fjågesund (2018).

Figura 1

Esquema Típico de Programa de Revestimento

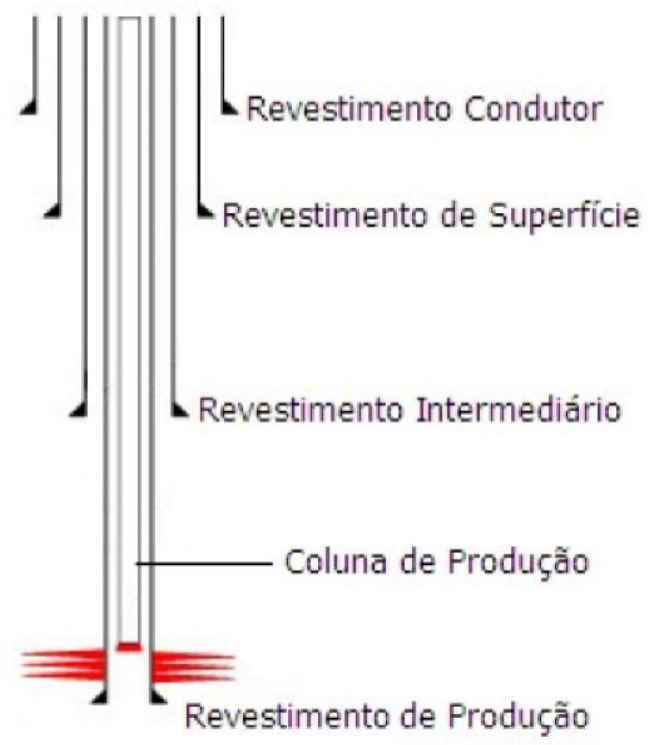

Fonte: Costa (2016).

Espera-se que em uma seção do revestimento, os tubos possuam as mesmas especificações técnicas, como: grau, peso linear, diâmetro externo, espessura e tipo de conexão. Roque (1992) não 
recomenda um número muito grande de seções devido a uma série de problemas operacionais e afirma ainda que esse número vai depender do comprimento da coluna, da logística e da análise de custos.

Um programa de revestimento deve atender às mínimas condições necessárias de viabilidade operacional e financeira do poço, sendo capaz de garantir segurança nas etapas de perfuração, completação, workover (intervenção) e produção, com mínimo custo possível (Correia \& Santos, 2017).

Um projeto típico de revestimento deve conter parâmetros como o número de fases e seus respectivos diâmetros, a profundidade de assentamento das sapatas e a seleção das especificações técnicas dos tubos de revestimentos, além da definição da composição e quantidade de seções das colunas. A seleção destes parâmetros de projeto é função de inúmeros fatores, tais como as condições geológicas, proteção de aquíferos, finalidade do poço, condições do reservatório, número e comprimento dos tubos de produção, tipos de elevação artificial, regulamentações governamentais, entre outros, conforme discutido por Ernens et al. (2018) e Wilson (2018). Além desses fatores, devese destacar que o projeto deve fornecer um poço executável e seguro às instalações, ao meio ambiente e às vidas humanas envolvidas.

O presente artigo propõe-se a desenvolver uma estratégia de otimização de seleção de revestimento de poço, combinando a avaliação de integridade mecânica de revestimento por meio de análise triaxial de tensões em estado limite de serviço e técnicas de gerenciamento de seleção de revestimento com base no menor peso, menor custo e com base na técnica de mínimo custo global.

\section{Metodologia}

Para a verificação da segurança operacional de um poço, o Instituto Americano de Petróleo (American Petroleum Institute - API) disponibiliza normas que auxiliam as operadoras na seleção dos tubos e conexões, bem como também na fabricação e manuseios deles. As Principais normas API são: (1) API TR 5C3 (2008), que determina o método de cálculo das resistências mecânicas; (2) API SPEC 5B (2008), que especifica as conexões entre os tubos e (3) API SPEC 5CT (2010), que especifica tubos e conexões.

Para se avaliar a resistência a partir de um dimensionamento tridimensional geralmente é empregado o Critério de Falha de von Mises (Teoria da Máxima Energia de Distorção). Para se obter a solução do problema, assume-se que a tensão triaxial equivalente de von Mises é igual à tensão de escoamento do material $\left(Y_{p}\right)$, admitindo que o escoamento se inicia da superfície interna do tubo. Feito isso, chega-se a soluções para a pressão de colapso e pressão de burst, que são mostradas nas equações 1 e 2 .

$$
P_{\text {colapso }}=-\frac{\left(\sigma_{z}+\sqrt{4 Y_{p}^{2}-12 \tau_{h a}^{2}-3 \sigma_{z}^{2}}\right)\left(D^{2}-d^{2}\right)}{4 D^{2}}
$$




$$
P_{\text {burst }}=\frac{d^{2} \sigma_{z}+\sqrt{3 D^{2} Y_{p}^{2}-9 D^{4} \tau_{h a}^{2}-3 D^{4} \sigma_{z}^{2}+Y^{2} d^{4}-3 d^{4} \tau_{h a}^{2}}\left(D^{2}-d^{2}\right)}{3 D^{4}+d^{4}}
$$

A pressão de colapso (Eq. 1) é empregada nas situações em que o nível de pressão externa atuando no tubo supera a pressão interna no tubular, provocando ovalização ou até instabilidade na seção transversal do revestimento. Já a expressão de pressão interna, burst, (Eq. 2) é empregada quando os níveis de pressão interna no corpo do revestimento são superados pela pressão interna, tendendo a um efeito de expansão da geometria interna, também conhecido por efeito balão. O equacionamento depende de propriedades geométricas do corpo do tubo (diâmetro externo $D$, diâmetro interno $d$ ) e dos campos de tensões envolvidos (tensão de cisalhamento $\tau_{h a}$ e tensão axial $\left.\sigma_{z}\right)$

As equações correspondem, respectivamente, às partes inferiores e superiores do diagrama mostrado na Figura 2 (Elipse de Cargas Triaxiais), ao longo do eixo de força axial nula. Tal diagrama ainda é completado pela envoltória API - que é desenvolvida a partir das especificações da norma API TR 5C3 (2008) e de fatores de segurança, para garantir uma maior segurança de projeto.

O método de análise triaxial (Fig. 2) permite a avaliação simultânea da integridade do revestimento com base nos cenários críticos de esforços que o sistema de revestimento deve suportar. Os esforços atuantes ao longo da profundidade do revestimento são representados graficamente por pares ordenados (esforço axial, pressão diferencial). Para garantia de atendimento de integridade estrutural do sistema, os valores plotados dos pares ordenados (esforço axial, pressão diferencial) devem estar contidos no espaço delimitado pela envoltória da elipse de von Mises e pela envoltória de segurança API, conforme detalhado na Figura 2. 
Figura 2

Elipse de Resistência Triaxial para Tubos de Revestimento API

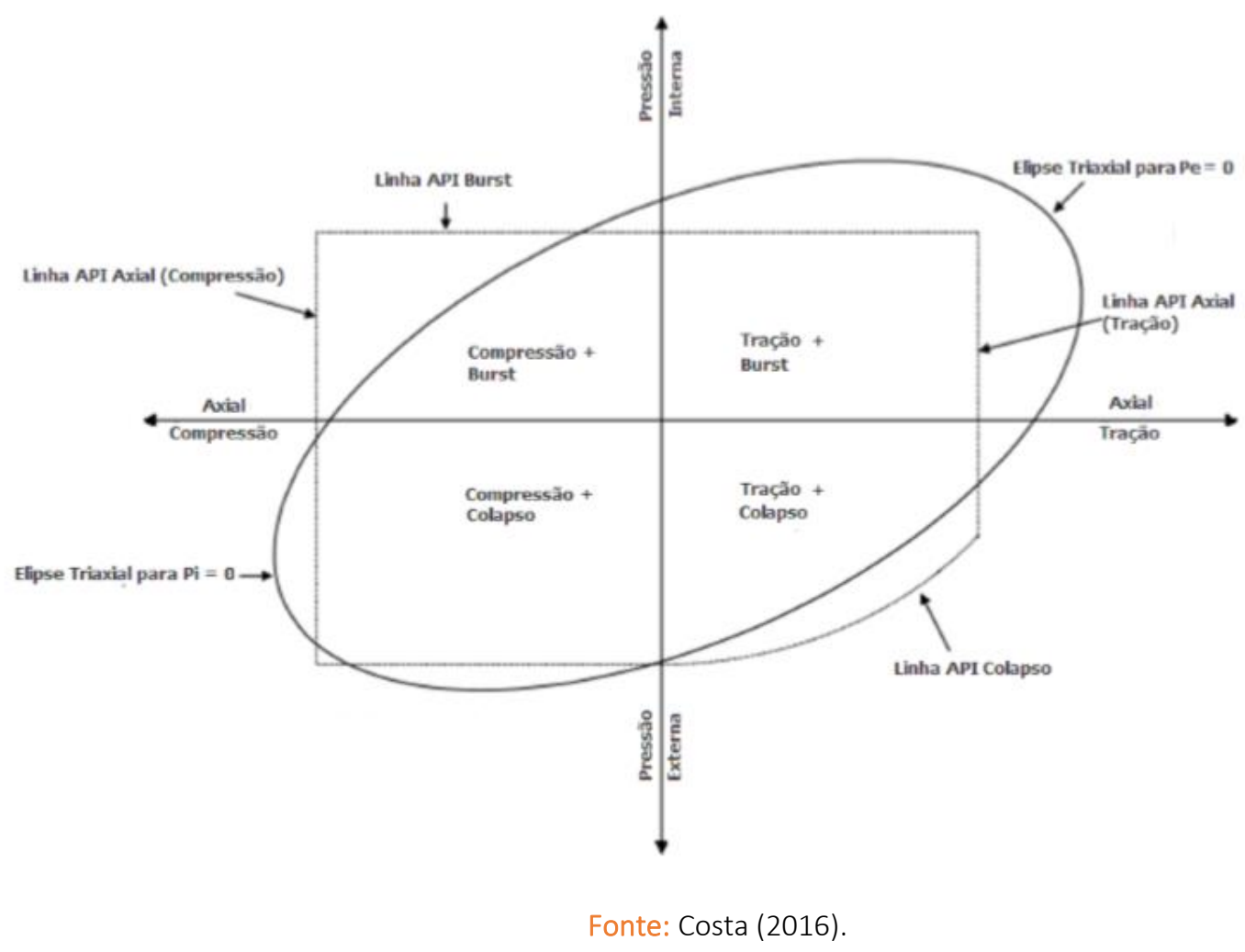

Portanto, uma vez conhecidos os esforços que hão de ser suportados pelo revestimento, podese iniciar a análise de qual material irá suportar tal esforço, para então daí ser iniciado um procedimento de otimização da escolha do revestimento - seja a partir do mínimo peso, mínimo custo ou mínimo custo global.

Ao longo do tempo, diversos autores desenvolveram pesquisas que abordam o problema da otimização da seleção de tubos, seja para se determinar que a coluna de revestimento possua um menor peso, ou para que ela tenha um menor custo, assim se enquadrando nas condições encontradas no poço, conforme pode ser visto nos trabalhos desenvolvidos por Huang et al. (2018), Khosravanian \& Aadnoy (2016) e Anon (2000).

Segundo Roque (1992), os métodos resultantes desses estudos podem ser agrupados em: (1) Método do Mínimo Peso; (2) Método do Mínimo Custo e (3) Método do Mínimo Custo Global.

Tais métodos foram desenvolvidos de forma que possam funcionar separadamente dos programas de cálculo das tensões que atuam no revestimento, de forma que possam auxiliar e completar tais métodos, realizando a otimização da escolha do revestimento. 
Mínimo peso

O método do mínimo peso baseia-se na seleção do revestimento de menor custo final dentre os mais leves capazes de suportar os esforços máximos previstos.

Segundo Roque (1992), a prioridade é dada ao peso em relação ao preço. Neste método, a coluna é discretizada em comprimentos mínimos de seção $L_{n}$. Para cada seção são selecionados os tubos de menor peso que atendam às exigências requeridas pelo problema, e dentre estes tubos selecionados se seleciona o de menor preço. Matematicamente, o custo total da coluna de menor peso pode ser encontrado pelo conjunto de equações 3, 4, 5 e 6 .

$$
\begin{gathered}
C_{T \min }=\min \left[C_{T}\left(W_{T M I N}\right)\right] m \\
W_{T M I N}=\min \left(W_{T}\right)_{m} \\
W_{T}=K_{m \rightarrow f t} \sum_{n=1}^{R} L_{n} W_{n} \\
C_{T}=\sum_{n=1}^{R} L_{n} C_{n}
\end{gathered}
$$

Nessas relações, temos que $C_{T m i n}$ é o custo total da coluna de menor peso, em unidades monetárias. $C_{T}$ é o custo total, em unidades monetárias. $C_{n}$ representa o custo unitário, em unidades monetárias por metro. $W_{T M I N}$ é o peso da coluna de menor peso dentre as mais leves, em libras força. $W_{T}$ é o peso total da coluna, em libras força. $W_{n}$ é o peso unitário, em libras força por pé. $L_{n}$ é o comprimento da seção, em metros. $K_{m \rightarrow f t}$ representa o fator de conversão de metros para pés e corresponde a 3.28084. O número total de colunas com mínimo peso ou mínimo custo é representado por $m$. Já $R$ descreve o número de seções que compõem a coluna.

\section{Mínimo custo}

O método do mínimo custo baseia-se na seleção da coluna de menor custo dentre todas as colunas capazes de suportar as cargas máximas previstas. Esse método pode ser representado matematicamente pela equação 7 .

$$
C_{\text {Tmin }}=\min \left(\sum_{n=1}^{N} L_{n} C_{n}\right)_{m}
$$

Conforme discutido por Roque (1992), a existência dos métodos do Mínimo Peso e do Mínimo Custo se explica pelo fato de que não é possível se otimizar, simultaneamente, o peso total e o custo final de uma coluna de revestimento. 


\section{Mínimo custo global}

Neste método, os mesmos princípios dos métodos anteriores - Mínimo Peso e Mínimo Custo são utilizados. Ainda, a cada trecho analisado, se considera todos os tubos mais leves que o mais barato e todos os tubos mais baratos que o mais leve que possam suportar os esforços previstos previamente. Essa metodologia de seleção é esquematizada pela Figura 3.

\section{Figura 3}

Critério de Seleção de Tubos do Método Mínimo Custo Global

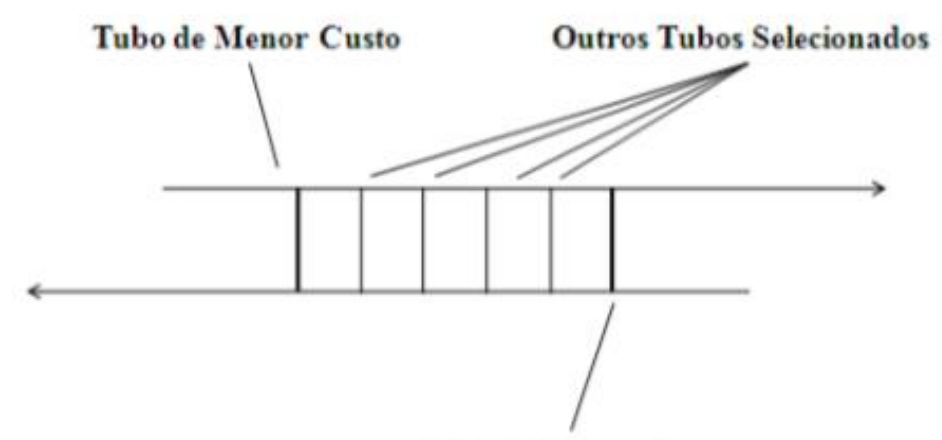

Tubo de Menor Peso

Fonte: Roque (1992).

Segundo Roque (1992), esse método busca a hipótese de uma coluna que suporte os máximos esforços previamente calculados cujo preço final seja menor que o determinado pelo Método do Mínimo Custo. Essa hipótese é justificada pelo fato de que os tubos determinados pelo Método do Mínimo Custo podem ser mais pesados. Por consequência, se os tubos mais pesados são utilizados na porção inferior da coluna, isso causa maior esforço axial nas seções superiores, induzindo o uso de tubos mais nobres nas seções superiores para resistir a esse esforço adicional.

A Figura 4 ilustra a evolução do custo acumulado para cada um dos três métodos, supondo que haja uma solução para o Método do Mínimo Custo Global. Vale ressaltar que, por atender simultaneamente as funções objetivo de minimização de peso (Eq. 3) e minimização de custo (Eq. 7), o Método do Mínimo Custo Global teoricamente deve retornar os valores ótimos entre as possíveis combinações das técnicas de otimização discutidas. 


\section{Figura 4}

\section{Conflito Custo/Peso}

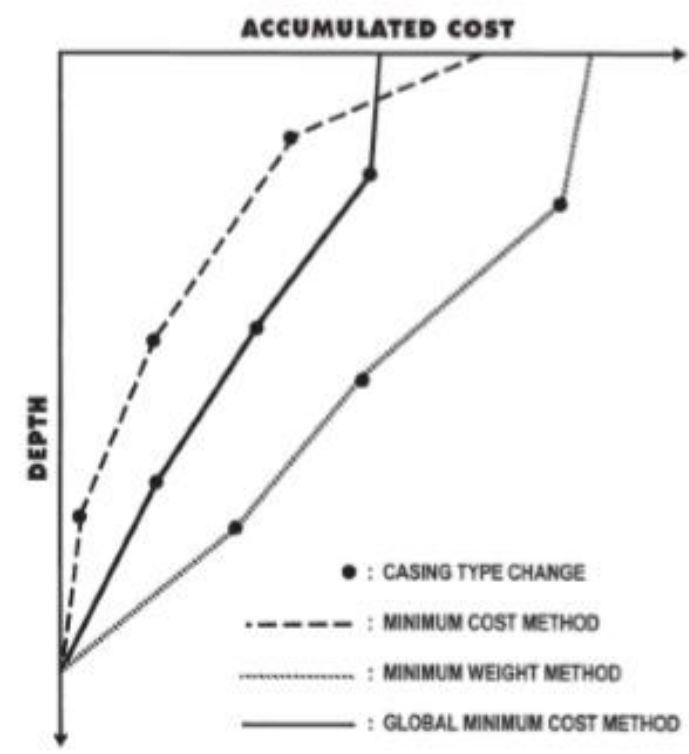

Fonte: Roque (1992)

No presente trabalho é proposta a junção das metodologias de análise de integridade mecânica por meio de análise triaxial acoplada aos modelos de seleção de revestimento, de forma a obter configurações ótimas de projetos de revestimentos. Para auxiliar a aplicação desta metodologia, juntamente com o seu desenvolvimento foi construído um sistema de interface gráfica que será incorporado ao aplicativo CWELL - que faz o dimensionamento automatizado dos revestimentos, o qual compõe o Sistema de Aplicações de Engenharia de Petróleo (SAEP), desenvolvido pela Universidade Federal de Alagoas por Costa (2016). Tal interface é construída em Python, com auxílio de Framework Django, sendo seu front-end desenvolvido em linguagem HTML (HyperText Markup Language), CSS (Cascading Style Sheets) e JavaScript. Mais detalhes sobre a implementação desse tipo de Framework podem ser vistos em Minetto (2007).

A seleção e a análise do desempenho de cada seção seguem o fluxograma mostrado na Figura 5. A análise consiste em verificar a estabilidade estrutural de cada seção diante dos esforços previstos. As resistências dos tubos são calculadas em concordância com a norma API TR 5C3 (2008). As cargas de colapso devidas ao cenário de perda total de circulação e as cargas de burst associadas a um teste de pressão após cimentação são calculadas na base das seções (ponto mais crítico). Para as cargas de tração tomou-se o valor crítico no topo de cada seção. 
Figura 5

Fluxograma da Seleção do Revestimento de Produção

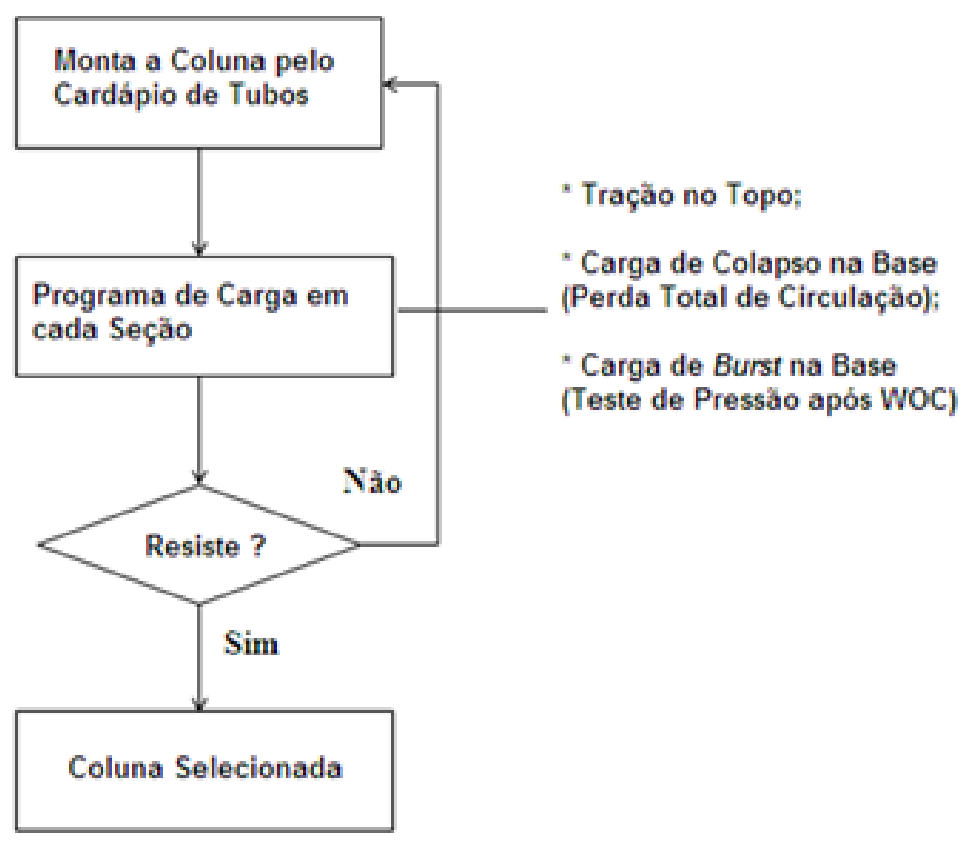

Fonte: Elaboração dos autores.

\section{Resultados e discussões}

A fim de ilustrar a potencialidade da metodologia proposta para otimização de seleção de revestimento de poço, é avaliado um estudo de caso de um poço vertical onshore, perfurado em três fases (revestimentos condutor, intermediário e de produção), como descrito na Tabela 1. Para este estudo os fatores de segurança utilizados foram: 1.3 (tração/compressão), 1.1 (burst), 1.0 (colapso) e 1.25 (triaxial).

Tabela 1

Programa de Revestimento

\begin{tabular}{lllll} 
Fase & Base $(\mathbf{m})$ & TC & $D_{\text {poco }}($ in) & $\rho_{m}(\mathbf{l g} / \mathrm{gal})$ \\
\hline Condutor - 20", H40, 94 lb/ft & 400 & Todo & 26 & 9.5 \\
Intermediário - 13 3/8", K55, 61 lb/ft & 1400 & Todo & 17.5 & 10 \\
Produção - 7", C75, 32 lb/ft & 2400 & Metade & 8.5 & 12 \\
\hline
\end{tabular}

Fonte: Elaboração dos autores.

Em todos os cenários será adotada uma espessura mínima de $87.5 \%$ da espessura nominal. Dessa forma, a espessura utilizada no revestimento condutor é de 0.438 in, no revestimento 
intermediário, de 0.430 in e no revestimento de produção, de 0.453 in. Em todas as fases, a velocidade de descida dos revestimentos é de $5 \mathrm{ft} / \mathrm{s}$ e a pasta de cimento é bombeada a uma pressão de 1000 psi com uma massa específica de $16 \mathrm{lb} / \mathrm{gal}$. Após seu endurecimento, tempo que é definido pela sigla WOC (Waiting on Cement), idealiza-se que a massa específica cai para $9 \mathrm{lb} / \mathrm{gal}$.

A Tabela 2 apresenta os cenários a serem avaliados em cada fase de estudo do programa de revestimento. Espera-se que na perfuração da fase de produção o gradiente de pressão da formação seja de $10.87 \mathrm{lb} /$ gal e o gradiente do influxo de gás seja de $0.33 \mathrm{psi} / \mathrm{m}$.

\section{Tabela 2}

Cenários do Programa de Revestimento

Fase

Burst

\section{Colapso}

$\begin{array}{ccc}\text { Condutor } & \text { Teste de Pressão após WOC }\left(P_{\text {teste }}=1200 \mathrm{psi}\right) & \text { Cimentação } \\ \text { Intermediário } & \text { Poço Completo de Gás } & \text { Perda Parcial de Circulação } \\ \text { Produção } & \text { Teste de Pressão após WOC }\left(P_{\text {teste }}=3500 \mathrm{psi}\right) & \text { Perda Total de Circulação }\end{array}$

Fonte: Elaboração dos autores.

A análise otimizada na montagem das seções do revestimento aplicou-se à última fase do revestimento, ou seja, no revestimento de produção. A figura 6 apresenta os resultados da análise de integridade mecânica, indicando que o tubo analisado suporta adequadamente aos níveis de carregamentos impostos no sistema. 
Figura 6

Resistências Triaxiais + Cenários do Revestimento de Produção

C75, 7 (in), 32 (lb/ft), 0.453 (in), $0.0-2400.0$ (m)

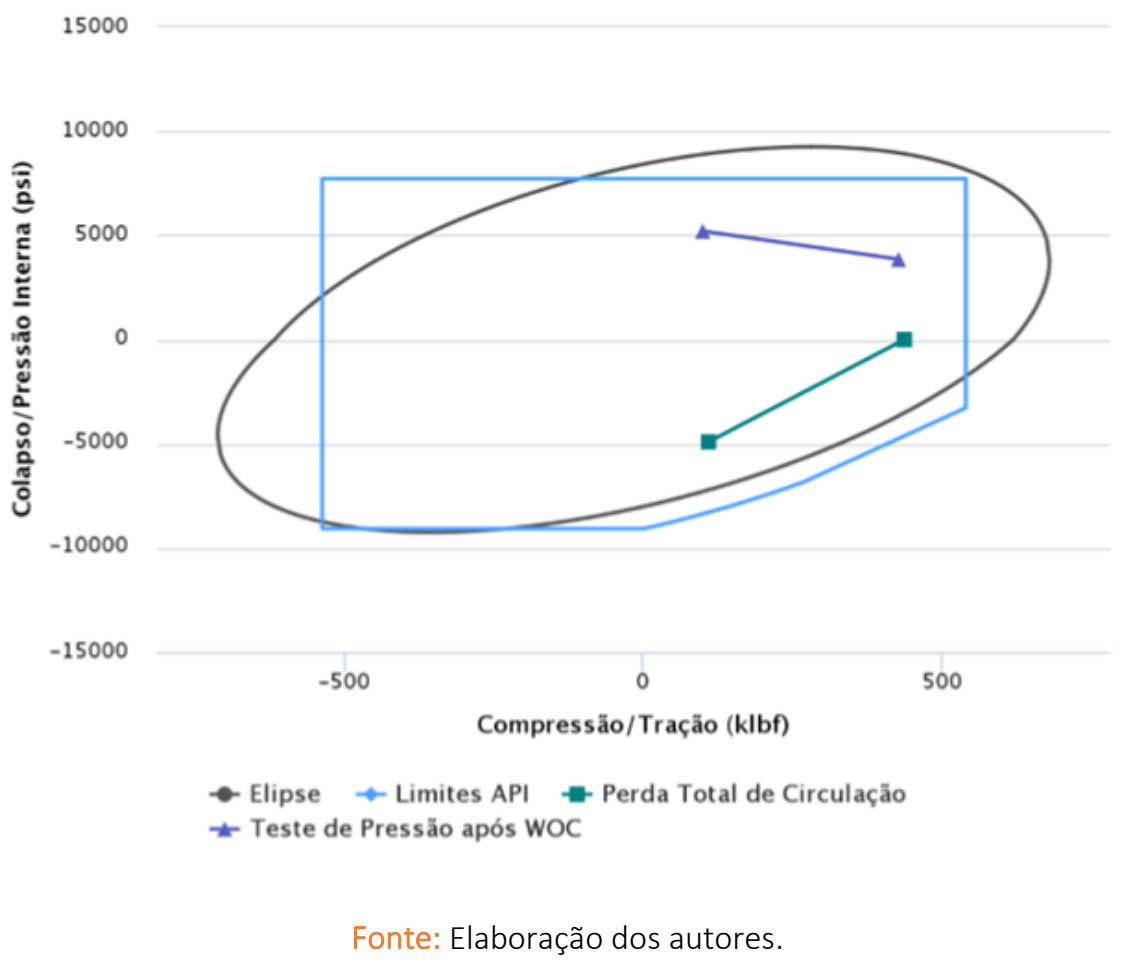

Tomando a Tabela 3 como base, pode-se concluir que o tubo de 7", de grau C75 e peso $32 \mathrm{lb} / \mathrm{ft}$, utilizado na coluna de revestimento de produção do estudo anterior, e assentado a uma profundidade de 2400 m, tem peso total de 251,968.512 lbf e custo total de U\$ 195,960.00. Para obtenção de uma coluna ótima, tomaram-se 3 seções, número máximo de tubos com propriedades distintas. Adotaramse comprimentos iguais para as seções, logo, o comprimento individual é de $800 \mathrm{~m}$.

\section{Tabela 3}

Preço de Tubos de 7"de Diâmetro

\begin{tabular}{|cccc|}
\hline Grau & Peso (lb/ft) & Espessura (in) & Custo (U\$/m) \\
\hline J55 & 23 & 0,317 & 40,58 \\
K55 & 23 & 0,371 & 44,07 \\
C95 & 23 & 0,317 & 55,58 \\
C75 & 23 & 0,317 & 61,44 \\
J55 & 26 & 0,362 & 43,56 \\
K55 & 26 & 0,362 & 49,09 \\
N80 & 26 & 0,362 & 55,57 \\
C95 & 32 & 0,453 & 77,33 \\
C75 & 32 & 0,453 & 81,65 \\
\hline
\end{tabular}

Fonte: Elaboração dos autores. 
As respostas obtidas pelos métodos do mínimo peso e do mínimo custo são apresentadas nas Tabelas 4 e 5, indicando assim a coluna de menor peso e a de menor custo, respectivamente.

Tabela 4

Coluna de Menor Peso

\begin{tabular}{cccc} 
Grau & Peso $(\mathrm{lb} / \mathrm{ft}) \mid$ Espessura $($ in $)$ & BASE $(\mathrm{m}) \mid \mathrm{TOPO}(\mathrm{m})$ & Preço $(\mathbf{U} \$ \mathbf{m})$ \\
\hline J55 & $23.0 \mid 0.317$ & $800 \mid 0$ & 40.58 \\
N80 & $23.0 \mid 0.317$ & $1600 \mid 800$ & 49.41 \\
C95 & $26.0 \mid 0.362$ & $2400 \mid 800$ & 62.82 \\
& Peso Total $=188,976.384 \mathrm{lbf}$ & & \\
& Custo Total $=$ U $\$ 122,248.00$ & & \\
\hline
\end{tabular}

Fonte: Elaboração dos autores.

Conforme resultados apresentados na tabela 4, o cenário ótimo considerando o modelo de otimização baseado em menor peso consiste no uso de 3 seções de revestimento, onde nos primeiros $800 \mathrm{~m}$ devem ser empregados tubos de grau J55, no trecho de $800 \mathrm{~m}$ a $1600 \mathrm{~m}$ deve-se empregar os tubos da classe N80 e no trecho de 1600 a 2400 recomenda-se o uso de tubos de grau C95, perfazendo custo total de $U \$ 122,2480.00$.

No entanto, a partir da aplicação da técnica de otimização baseada no menor custo, conclui-se que a combinação deve envolver apenas dois tipos de revestimento: nos primeiros $1600 \mathrm{~m}$ deve-se empregar o tubo de grau J55, enquanto que no trecho final, na profundidade de $1600 \mathrm{~m}$ a $2400 \mathrm{~m}$ devese fazer uso dos tubos de grau N80, conforme destacado na tabela 5, perfazendo um custo total de U\$ $111,768.00$.

Tabela 5

Coluna de Menor Custo

\begin{tabular}{cccc} 
Grau & Peso $(l b / f t) \mid$ Espessura $($ in $)$ & BASE $(\mathrm{m}) \mid$ TOPO $(\mathrm{m})$ & Preço $(\mathbf{U} \$ \mathbf{m})$ \\
\hline J55 & $23.0 \mid 0.317$ & $800 \mid 0$ & 40.58 \\
J55 & 26.010 .362 & $1600 \mid 800$ & 43.56 \\
N80 & $26.0 \mid 0.362$ & $2400 \mid 1600$ & 55.57
\end{tabular}

Peso Total $=196,850.40 \mathrm{lbf}$

Custo Total $=$ U $\$ 111,768.00$

Fonte: Elaboração dos autores.

Conclui-se que utilizando a otimização do mínimo peso, a coluna de revestimento de produção 
com três seções teria uma redução de custos de $19,92 \%$, com os valores sendo reduzidos de U\$ $150,768.00$ para a casa de $U \$ 122,240.00$.

A partir dos resultados obtidos com a técnica de otimização baseada no mínimo custo, a redução seria de 16,19\%, partindo de U\$133,368.00 para U\$111,768.00.

Os resultados foram comparados aos estudos desenvolvidos por Roque (1992), obtendo convergência nos valores apresentados. Os resultados de Roque (1992) empregam metodologia similar, porém a partir de formulação desacoplada com maior dispêndio computacional, ao invés da combinação de otimização com a análise triaxial proposta no presente trabalho.

\section{Considerações finais}

A metodologia desenvolvida apresentou versatilidade nas estratégias implementadas, podendo ser empregada no processo de dimensionamento de colunas de revestimento de poços de petróleo, servindo de apoio aos projetistas e para fins educacionais.

A aplicação de técnicas de gestão de projetos de revestimento de poço, integrando a otimização econômica e a garantia de integridade estrutural do sistema correspondem ao estado da arte no campo de sistemas de revestimento de poços de petróleo.

A abordagem de técnicas de otimização evidenciou que o projeto de revestimento de poços pode ter elevadas economias nos processos de seleção de parâmetros de projeto de revestimento. No caso analisado, a combinação de seções por meio da técnica de otimização revelou economia de até $19,92 \%$ dos custos envolvidos.

Vale ressaltar que os resultados de benefícios econômicos obtidos são dependentes do cenário de carregamento específico analisado. No caso apresentado avaliou-se a integridade do revestimento de produção em um cenário de perda total de circulação durante a perfuração do poço em questão. Para avaliação final da economia global a ser obtida, sugere-se em trabalhos futuros a análise de diversos possíveis cenários de falha, onde a avaliação econômica deve ser feita com base no cenário de integridade estrutural mais crítico.

\section{Referências}

Aadnoy, B. (2010). Modern Well Design. 2nd. ed. Leiden, The Netherlands: CRC Press.

Anon. (2000). Multistring-casing-design optimization with wellhead growth. JPT, Journal of Petroleum Technology, 52(5), 49.

American Petroleum Institute (2008). API TR 5C3: Specification for Threading, Gauging and Thread Inspection of Casing, Tubing, and Line Pipe Threads. Fifteenth Edition. 
Correia, J.P.A., Santos, J.P.L. (2017). Avaliação numérica da integridade estrutural de revestimento de poços de petróleo em zonas de alta pressão e alta temperatura e áreas de rochas salinas. Revista Holos, Vol. 1, Pag. 292-305. doi http://dx.doi.org/10.15628/holos.2017.5178.

Costa, J. H (2016). Sistema de aplicações de engenharia de petróleo (SAEP): módulo poço.. Trabalho de Conclusão de Curso - Universidade Federal de Alagoas, Maceió.

Ernens, D., Hariharan, H., van Haaften, W. M., Pasaribu, H. R., Jabs, M., \& McKim, R. N. (2018, September 1). Improving Casing Integrity With Induction Brazing of Casing Connections. Society of Petroleum Engineers. https://doi.org/10.2118/184703-PA.

Ezeakacha, C. P., Salehi, S., \& Kiran, R. (2018). Lost circulation and filter cake evolution: Impact of dynamic wellbore conditions and wellbore strengthening implications. Journal of Petroleum Science and Engineering, 171, 1326-1337. https://doi.org/10.1016/j.petrol.2018.08.063.

Fjågesund, T. (2018, December 1). Guest Editorial: Robust Well Barrier and Well Integrity Management. Society of Petroleum Engineers. https://doi.org/10.2118/1218-0014-JPT

Huang, W., Gao, D., \& Liu, Y. (2018). Mechanical model and optimal design method of tubular strings with connectors constrained in extended-reach and horizontal wells. Journal of Petroleum Science and Engineering, 166, 948-961. https://doi.org/10.1016/j.petrol.2018.01.072.

Khosravanian, R., \& Aadnoy, B. S. (2016). Optimization of casing string placement in the presence of geological uncertainty in oil wells: Offshore oilfield case studies. Journal of Petroleum Science and Engineering, 142, 141-151. https://doi.org/10.1016/j.petrol.2016.01.033.

Liu, K., Gao, D., \& Taleghani, A. D. (2018). Analysis on integrity of cement sheath in the vertical section of wells during hydraulic fracturing. Journal of Petroleum Science and Engineering, 168, 370379. https://doi.org/10.1016/j.petrol.2018.05.016.

Minetto, E. L. (2007) Frameworks para Desenvolvimento em PHP. 1. Ed. São Paulo: Novatec,. p. 17-19.

Roque, J. L. (1992). Dimensionamento de Revestimentos para Poços Profundos, Poços Direcionais e Horizontais de Longo Afastamento Horizontal Pelo Método do Mínimo Custo Global. 1992. 114 f. Dissertação (Mestrado em Engenharia de Petróleo) - Faculdade de Engenharia Mecânica, Universidade Estadual de Campinas - UNICAMP.

Wilson, A. (2018, June 1). Risk-Based Statistical Approach To Predict Casing Leaks. Society of Petroleum Engineers. https://doi.org/10.2118/0618-0071-JPT.

Xia, J., Li, K. X., Ma, H., \& Xu, Z. (2015). Joint planning of fleet deployment, speed optimization, and cargo allocation for liner shipping. Transportation Science, 49(4), 922-938. https://doi.org/10.1287/trsc.2015.0625. 